\title{
Treatment Options for Sciatica: Surgery, Epidural Injections, and Osteopathic Manipulative Treatment
}

\author{
Roya Vahdatinia ${ }^{1}$, Leonard B Goldstein ${ }^{2 *}$, Victoria Troncoso ${ }^{3}$ and David Shoup ${ }^{3}$ \\ ${ }^{1}$ School of Osteopathic Medicine, AT Still University, United States \\ ${ }^{2}$ Clinical Education Development, AT Still University, United States \\ ${ }^{3}$ Department of Osteopathic Principles and Procedures, AT Still University, United States
}

*Corresponding author: Leonard B Goldstein, Assistant Vice President for Clinical Education Development, AT Still University, 5850 E Still Circle, Mesa, AZ 85206 , United States, Tel: 4802196195; Email: Igoldstein@atsu.edu

Received date: February 10, 2017; Accepted date: February 21, 2017; Published date: March 05, 2017

Copyright: (c) 2017 Vahdatinia R, et al. This is an open-access article distributed under the terms of the Creative Commons Attribution License, which permits unrestricted use, distribution, and reproduction in any medium, provided the original author and source are credited.

\begin{abstract}
Understanding the etiology and optimal treatment options for sciatica is a frequently discussed medical topic. This study analyzes treatment options-specifically surgery, epidural steroid injections (ESI), and osteopathic manipulative treatment (OMT)-for sciatic radiculopathy due to lumbar disc herniation. A review of several studies comparing surgery to non-surgical intervention, surgery to ESI, ESI to conservative care, and OMT is discussed. There is no consensus on the usefulness of ESI; several studies illustrated efficacy while numerous others did not conclude statistical significance, and rather, highlighted the adverse effects. In contrast, OMT is considered a non-invasive first-line approach to achieving relief from sciatica, while surgery is a last resort in the uncomplicated patient.
\end{abstract}

Keywords: Treatment options; Sciatica; Surgery; Epidural injections; Osteopathic manipulative treatment

\section{Introduction}

The sciatic nerve, formed by the L4-S3 nerve roots, passes through the sciatic foramen usually inferior to the piriformis muscle before descending the posterior thigh. If compressed or irritated, it can lead to pain, numbness, and/or paresthesias which may radiate from the posterior buttock and even to the foot [1]. Proximal to the popliteal fossa, the sciatic nerve divides into the tibial and common fibular (peroneal) nerves [2]. The term sciatica is used broadly and can have multiple etiologies, with the most common being compression secondary to disc herniation and degenerative changes of the spine [1]. The most common sites of disc herniation are at the L4-L5 level and L5-S1 level, with L3-L4 herniations occurring more frequently with increasing age [3]. Other causes of sciatic pain include piriformis syndrome, foraminal stenosis, spondylolisthesis (usually involving anterior slippage of L5 over S1), pelvic floor tumors, obstetrical compression, synovial cysts, and trauma from gluteal injections [1]. In order to effectively treat sciatic pain, the etiology must first be determined; this review will discuss different treatment approaches including surgery, epidural injections, and osteopathic manipulative treatment (OMT) to treat sciatica due to lumbar disc herniation.

There has been multiple studies conducted comparing surgery to non-surgical intervention. One randomized trial with $n=283$ and a low risk of bias compared patients suffering from sciatica for 6 weeks to 12 weeks who underwent early microdiscectomy (mean of 2 weeks) versus those who followed prolonged conservative treatment. The patients who underwent early surgery reported faster relief of leg pain at 3 months and had a median recovery time of 4 weeks (versus 12.1 weeks in the conservative treatment group) though at 1 and 2 years follow-up, there were no differences between the two groups. In another study with $n=501$, after 2 years, both the conservative group and surgery group improved in regards to primary (leg and/or back pain, perceived recovery, leg pain specific functional status, lost days at work) and secondary outcomes with no statistically significant differences [4].

In comparing surgery to epidural steroid injections (ESI), one study compared leg pain in patients who received ESIs versus those who underwent microdiscectomy. At 3 months and 6 months follow-up intervals, there was a statistically significant decrease in leg pain in the surgical group. Approximately half of the participants (27) who received ESI crossed over to the surgical group and experienced similar outcomes of relief in leg pain. However, the high risk of bias of this study makes it difficult to establish a consensus between microdiscectomy to ESI [4].

The argument for epidural injections is that sciatic pain is predominantly due to inflammation rather than mechanical compression [5]. Bhatti et al. conducted a meta-analysis of different epidural injection approaches including lumbar transforaminal epidural injection (TEI), lumbar interlaminar epidural injection (IEI), and caudal epidural injections (CEI) and their effects on chronic sciatica. In 16 studies compiled from 2006 to 2016, pain was assessed using the Numeric Rating Scale (NRS), Visual Analogue Scale (VAS), Verbal Numerical Rating Scale (VNRS) and Japanese Orthopedic Association (JOA). In 7 of the studies, pre-injection pain score using the NRS scale was 7.7 to 8.25 compared to 1.3 to 4.1 post-injection, showing a $50 \%$ to $83 \%$ improvement. Another 7 studies used the VAS scale, with one showing $83 \%$ improvement and another with $50 \%$ improvement in pain level. In one study, $68 \%$ of patients reported pain relief, though the results were not statistically significant [5].

Apart from pain scores, functional disability scoring was also analyzed for patients receiving ESI. Nine studies used the Oswestry Disability Index (ODI), 5 of which had an "ODI mean baseline value of 29 [which] reduced to 14 at the last follow-up, indicating a $>50 \%$ improvement in the functional disability score." All the studies 
reported for reduced disability in ODI, with the lowest being 26\% improvement after ESI [5].

In regards to the need for surgery post ESI, there was a rate of $13 \%$ to $21 \%$ in 7 studies. One study showed a $44 \%$ rate of surgery post ESI, while another showed only $7.05 \%$. "On average, $80 \%$ of patients were able to avoid surgical intervention with the help of EI treatment [5]."

Of the three approaches to epidural injections, transforaminal was the most common, probably because it targets a specific area. The interlaminar approach is useful for bilateral pain and the caudal approach can be used in areas of previous surgery and does not carry the risk of dural puncture [5]. Side effects of corticosteroid injections include spinal headache, tendon rupture, post-injection flare, tissue atrophy, and transient hyperglycemia. Female specific side effects include abnormal vaginal bleeding, decrease in lactation, facial flushing, and transient hirsutism [6]. More serious adverse effects include adrenal insufficiency, neurotoxicity (from inadvertent intrathecal injection), epidural abscess, fungal or bacterial injections, and paralysis [7].

In another analysis of epidural corticosteroid injections for the treatment of sciatica, there was no demonstrated efficacy among hundreds of patients. Compared to placebo, there was minimal relief at 3 months (shown in 23 randomized trials). Sciatic pain and disability was evaluated on a scale of 0 to 100 with patients reporting a mean improvement of 3 to 6 points among the different trials (with 10 or more points be clinically significant). Long term assessment (one year or more) also failed to demonstrate statistical significance in pain or disability between corticosteroid injections and placebo, based on a review of seven trials with $n=714$ [7].

Apart from surgery and epidural injections, osteopathic manipulative treatment (OMT) offers a more conservative and noninvasive approach. Because of this, OMT should be considered in early management. Though the effects of OMT on sciatic pain have yet to be clearly demonstrated in controlled studies, there is a plethora of supporting clinical evidence. An osteopathic treatment begins with a structural examination of the soft-tissue regions of the thoracic spine, lumbar spine, and pelvis, followed by a hands-on treatment to help relieve tissue tension, improve lymphatic drainage, and reduce inflammatory compression on the disc.

One such osteopathic treatment uses a modality referred to as counterstain which is a positional technique that facilitates relaxation of hypertonic muscles. Counterstain acts on the stretch reflex of the muscle to reset the feedback loop extending from the muscle to the dorsal horn of the spinal cord and then back to the muscle, which if activated causes the muscle to contract and remain in a spasm.

A common muscle to be hypertonic in sciatica is the iliopsoas which may result in concurrent Psoas Syndrome. Patients presenting with Psoas Syndrome usually present with flexed forward posturing, difficulty standing fully erect, pain in the lower back, and possible sciatic pain in the opposite leg. Other osteopathic treatment modalities to treat Psoas Syndrome includes muscle energy, still technique, and direct and indirect methods. High-velocity low-amplitude thrusting techniques are relatively contraindicated if neurologic signs are present. Another structure of clinical significance is the iliolumbar ligament, which emerges from the transverse processes of L4 and L5 and attaches to the iliac crest. Irritation of the iliolumbar ligament (due to a herniation) can refer pain to the groin, SI region, and lateral thigh. Structural examination should also include the ipsilateral adductor muscles which can also be restricted, and thus an additional area that would benefit from OMT [8].

Philip Greenman, Do, identified six areas of dysfunction that are commonly involved in low back pain, which he referred to as the dirty half dozen. Of the 183 patients with chronic low back pain who he examined, $50 \%$ had three or more of the six dysfunctions. The six dysfunctions included non-physiologic pelvic shears, sacral torsions, short leg, muscle imbalance (i.e. psoas syndrome), pubic symphysis shears, and dysfunctions of the thoracic and lumbar spine (non-neutral - sidebending and rotation of vertebral segments to the same side). When these were treated with OMT, it resulted in $75 \%$ of the group returning to work and/or activities of daily living [9].

In a study titled "Osteopathic Manipulative Treatment is Effective for Acute Low Back Pain in the Military," Cruser et al conducted a randomized, controlled trial with 60 soldiers aged 18 to 35 with low back pain (onset within 30 days). The participants either received usual care only (UCO), which included nonsteroidal analgesics, muscle relaxants, and physical therapy, while the OMT group received once weekly OMT for 4 weeks. The OMT protocol was designed to treat Dr. Greenman's dirty half dozen dysfunctions and was provided by 3 osteopathic physicians and 1 allopathic physician. Pain intensity using the Quadruple Visual Analog Scale (QVAS) and functioning ratings on the Roland-Morris Disability Questionnaire were assessed before and 4 weeks after the trial. The OMT group reported statistically significant post-trial pain $(p=0.026)$, lower pain intensity in "pain now" $(p=0.025)$ and "pain typical" $(\mathrm{p}=0.020)$ categories during all visits, and improvement of "pain at best" sooner $(\mathrm{p}=0.004)$, supporting the use of OMT for acute low back pain [10]. Though this study did not assess whether patients had MRI findings consistent with disc herniation, the results are promising for sciatica treatment due to herniation.

Although a comparison of OMT, epidural injections, and surgery has yet to be completed by one study, it can be concluded based on the meta-analysis of multiple separate studies that OMT is a first-line choice for treatment of sciatica, followed by epidural injections, with surgery as a last resort. In addition, the American College of Physicians (ACP) recently issued updated guidelines for treatment of low back pain (LBP). Though patients with both radicular and non-radicular symptoms were included in the systematic reviews which the guidelines are based upon, the outcomes evaluated included reduction or elimination in LBP, not specially a reduction in radicular LBP. Nonetheless, the first recommendation is based on the premise that most patients with acute or subacute LBP improve over time, regardless of treatment. It is recommended that first-line treatment include superficial heat, massage, acupuncture, or spinal manipulation. For patients desiring pharmacologic treatment, NSAIDs or muscle relaxants are recommended. For patients with chronic LBP (>12 weeks), the ACP recommendation is "treatment with exercise, multidisciplinary rehabilitation, acupuncture, mindfulness-based stress reduction, tai-chi, yoga, motor control exercise, progressive relaxation, electromyography biofeedback, low-level laser therapy, operant therapy, or spinal manipulation. [11]" When nonpharmacologic therapy is inadequate, patients can select NSAIDs as first-line and tramadol or duloxetine as second-line therapy, and opioids reserved for patients who have failed the aforementioned treatments [12].

OMT is a conservative approach of diagnosing soft tissue dysfunctions or restrictions followed by treatment aimed at restoring motion, improving fluid flow/drainage, and restoring tissue homeostasis. When considering the best treatment for a patient 
Citation: Vahdatinia R, Goldstein LB, Troncoso V and Shoup D (2017) Treatment Options for Sciatica: Surgery, Epidural Injections, and Osteopathic Manipulative Treatment. J Pain Manage Med 3: 122.

Page 3 of 3

suffering from sciatica, surgical intervention should be reserved when other options have been exhausted. Epidural injections may be a reasonable step in patients with disc herniation when more conservative treatments such as OMT have failed. A step-wise approach beginning with beneficial conservative treatments such as OMT is imperative for patients as options are discussed.

\section{References}

1. Longo DL, Allan HR, Ross DZ (2015) Sciatica. N Eng J Med 372 1240-1248.

2. Adibatti M, Sangeetha V (2014) Study on Variant Anatomy of Sciatic Nerve. 8: AC07-AC09.

3. Stafford MA, P Peng, DA Hill (2007) Sciatica: A Review of History, Epidemiology, Pathogenesis, and the Role of Epidural Steroid Injection in Management. Br J Anaesth 99: 461-473.

4. Jacobs WC, Van Tulder M, Arts M, Rubinstein SM, van Middelkoop M, et al. (2010) Surgery versus Conservative Management of Sciatica Due to a Lumbar Herniated Disc: A Systematic Review. Eur Spine J 20: 513-522.
5. Bhatti AB, Sunny K (2016) Role of Epidural Injections to Prevent Surgical Intervention in Patients with Chronic Sciatica: A Systematic Review and Meta-Analysis. Cureus: E723.

6. Brook EM, Hu CH, Kingston KA, Matzkin EG (2016) Corticosteroid Injections: A Review of Sex-Related Side Effects. Orthopedics: 1-5.

7. Sciatica and Epidural Corticosteroid Injections (2014) Rev Prescrire 37: 849-850.

8. Anthony GC (2011) Foundations of Osteopathic Medicine. 3rd ed. Philadelphia: Wolters Kluwer Health/Lippincott Williams \& Wilkins.

9. Kuchera ML (2005) Osteopathic Manipulative Medicine Considerations in Patients with Chronic Pain. J Am Osteopath Assoc 105: S29-S36.

10. Cruser DA, Douglas M, Kendi H, Sarah KB, Kathryn W, et al. (2012) A Randomized, Controlled Trial of Osteopathic Manipulative Treatment for Acute Low Back Pain in Active Duty Military Personnel. J Man Manip Ther 20: 5-15.

11. Qaseem A, Wilt TJ, McLean RM, Forciea MA (2017) Noninvasive Treatments for Acute, Subacute, and Chronic Low Back Pain: A Clinical Practice Guideline from the American College of Physcians. Ann Intern Med: 1-17. 